\title{
Interaction of Additive Noise and Nonlinear Dynamics in the Double-Gyre Wind-Driven Ocean Circulation
}

\author{
THEMISTOKLIS P. SAPSIS \\ Courant Institute of Mathematical Sciences, New York, New York \\ Henk A. DiJKstra \\ Institute for Marine and Atmospheric Research Utrecht, Department of Physics and Astronomy, Utrecht University, \\ Utrecht, Netherlands
}

(Manuscript received 12 March 2012, in final form 5 October 2012)

\begin{abstract}
In this paper the authors study the interactions of additive noise and nonlinear dynamics in a quasigeostrophic model of the double-gyre wind-driven ocean circulation. The recently developed framework of dynamically orthogonal field theory is used to determine the statistics of the flows that arise through successive bifurcations of the system as the ratio of forcing to friction is increased. This study focuses on the understanding of the role of the spatial and temporal coherence of the noise in the wind stress forcing. When the wind stress noise is temporally white, the statistics of the stochastic double-gyre flow does not depend on the spatial structure and amplitude of the noise. This implies that a spatially inhomogeneous noise forcing in the wind stress field only has an effect on the dynamics of the flow when the noise is temporally colored. The latter kind of stochastic forcing may cause more complex or more coherent dynamics depending on its spatial correlation properties.
\end{abstract}

\section{Introduction}

The barotropic vorticity equation forms the cornerstone model of the wind-driven ocean circulation. With the solutions of the linear part of this equation one can explain the origin of western boundary currents in the ocean (see e.g., Pedlosky 1987). The successive bifurcation behavior of solutions of the nonlinear equation has been actively studied, both for the single- (SG) and the double-gyre (DG) wind stress forcing (see Dijkstra 2005, chapter 5). The behavior of DG solutions of the barotropic vorticity equation in a square basin has been shown to display qualitatively similar features as those of shallow-water models in realistic basins. In this way, a relation can be established between different DG solutions and Kuroshio path transitions (Pierini et al. 2009) and the DG case is considered to be an important model

\footnotetext{
Corresponding author address: Henk A. Dijkstra, Institute for Marine and Atmospheric Research Utrecht, Dept. of Physics and Astronomy, Utrecht University, Princetonplein 5, 3584 CC Utrecht, Netherlands.

E-mail: h.a.dijkstra@uu.nl
}

for further theory development on the variability of (equivalent) barotropic ocean flows.

An important issue in the behavior of wind-driven flows is the effect of time-dependent wind forcing. The wind stress over the midlatitude oceans displays strict periodic components but otherwise is quite a largeamplitude noisy signal (Schmeits and Dijkstra 2001; Sura 2003). For the North Pacific, the spatial correlation scale is found to be about $1000 \mathrm{~km}$ and a typical decorrelation time scale is about one day (Sura 2003). The effect of additive, spatially coherent and temporally white noise on the behavior of the DG flows has been studied in a reduced gravity model (Sura et al. 2000, 2001). In a unique regime (relatively high friction), the low-frequency variability of the double-gyre flow can be understood as a red noise response (set 1 and set 3 in Sura et al. 2000). In a multiple equilibrium regime, the noise induces regime transitions and the spatial coherence of the noise appears crucial for these transitions to occur (Sura et al. 2001). In Sura and Penland (2002), it is shown that details in the stochastic forcing, in particular its temporal correlations, can have a significant effect on the statistics of regime transitions. Pierini (2010) also shows 
that a red noise wind forcing can induce low-frequency variability in a shallow-water model through a coherence resonance mechanism when the temporal decorrelation time scale is large enough. However, the precise role of wind stress noise in the path transitions of the Kuroshio current and the low-frequency variability of the Kuroshio Extension is still unclear.

The effect of additive noise in the wind stress forcing on the dynamics of the double-gyre flows has not been systematically studied because of the huge computational effort involved of solving the stochastic partial differential equations. Recently, however, new methods have become available that make such a study feasible. The dynamically orthogonal (DO) field method was developed to determine solutions to stochastic partial differential equations. It was applied to two-dimensional Navier-Stokes equations with a fixed number of modes in Sapsis and Lermusiaux (2009) and with an adaptive number of modes that vary according to the characteristics of the solution in Sapsis and Lermusiaux (2012) In the present paper we apply the DO method to the DG problem to study the effect of additive noise on the nonlinear DG flows. While the configuration (small basin, quasigeostrophic approximation) is strongly idealized, the results form the basis (just as those of the deterministic problem (Cessi and Ierley 1995; Dijkstra and Katsman 1997) to interpret results from more realistic models (and eventually of observations).

In section 2, the DG model and the DO methodology are shortly presented. Section 3 contains the results of the study of the effects of different types of noise on the statistics of the DG flows. We in detail analyze the effect of the spatial and temporal coherence on the statistical properties of the DG flows by determining the energy transfer between the different DO modes as well as the properties of the probability density functions. In this way, we provide links between the statistical responses of the system, its linear instabilities, and the effect of stochastic noise (with various properties) on the stabilization or destabilization of the flows. The results are summarized and discussed in section 4.

\section{Methodology}

To be self-consistent we give a brief summary of the barotropic DG model and the DO methodology (with additional details in the appendix).

\section{a. The barotropic double-gyre model}

Consider an idealized model of the midlatitude winddriven ocean ocean circulation consisting of water with constant density $\rho_{0}$ in an idealized square $(L \times L)$ ba$\sin$. The basin is located on a midlatitude $\beta$-plane with Coriolis parameter $f=f_{0}+\beta_{0} y$. The ocean flow is driven by a wind stress $\tau=\left(\tau^{x}, \tau^{y}\right)$ and characterized by a horizontal length scale $L$ and a horizontal velocity scale $U$. When the Rossby number $\epsilon=U /\left(f_{0} L\right)$ is small, quasigeostrophic theory is an adequate description of the large-scale flow (Pedlosky 1987). Let $\psi$ indicate the geostrophic streamfunction in the horizontal plane, then the vorticity $\zeta$ is given by and $\zeta=\nabla^{2} \psi$.

The governing equation in this theory is the barotropic vorticity equation, given by

$$
\begin{aligned}
\frac{\partial q}{\partial t}+J(\psi, q) & =A_{H} \nabla^{4} \psi+\frac{\nabla \times \boldsymbol{\tau}}{\rho_{0} H}, \quad \text { and } \\
q & =\nabla^{2} \psi+\beta_{0} y .
\end{aligned}
$$

Here, $q$ is the potential vorticity and the Jacobian operator $J$ is defined as $J(F, G)=F_{x} G_{y}-F_{y} G_{x}$ where the subscripts indicate differentiation. The quantity $A_{H}$ represents the turbulent lateral friction coefficient. Noslip boundary conditions are prescribed at the eastwest boundaries and slip conditions at the north-south boundaries, that is

$$
\begin{aligned}
& x=0, L: \psi=0, \quad \frac{\partial \psi}{\partial x}=0, \quad \text { and } \\
& y=0, L: \psi=0, \quad \frac{\partial^{2} \psi}{\partial y^{2}}=0 .
\end{aligned}
$$

The total wind stress is written as $\tau=\tau_{d}+\tau^{\prime}$, with $\tau^{\prime}$ indicating the stochastic component. The deterministic double-gyre wind stress profile $\tau_{d}$ considered with (1) is

$$
\tau_{d}^{x}(x, y)=-\tau_{0} \cos 2 \pi \frac{y}{L} ; \tau_{d}^{y}(x, y)=0,
$$

where $\tau_{0}$ is a typical amplitude.

Note that the Eqs. $(1 \mathrm{a}, \mathrm{b})$ can also be formulated in velocity $\left[\mathbf{v}=\left(v_{x}, v_{y}\right)\right]$ and pressure $(p)$ formulation as

$$
\begin{aligned}
\frac{\partial \mathbf{v}}{\partial t}+\mathbf{v} \cdot \nabla \mathbf{v}+f \mathbf{k} \times \mathbf{v} & =-\frac{1}{\rho_{0}} \nabla p+A_{H} \nabla^{2} \mathbf{v}+\frac{\tau}{\rho_{0} H}, \\
\nabla \cdot \mathbf{v} & =0
\end{aligned}
$$

where $\mathbf{k}$ is the unit vector in the $z$ direction. In this formulation, the boundary conditions become 


$$
\begin{aligned}
& x=0, L: v_{x}=v_{y}=0, \quad \text { and } \\
& y=0, L: v_{y}=\frac{\partial v_{x}}{\partial y}=0 .
\end{aligned}
$$

The velocity-pressure formulation will be used in the DO method as discussed in the next section.

\section{b. Dynamically orthogonal field equations}

If a general random state vector is denoted by $\mathbf{u}(x, y, t ; \omega)$, the starting point of the DO method is to use a generalized, time-dependent, Karhoenen-Loeve expansion

$$
\mathbf{u}(x, y, t ; \omega)=\overline{\mathbf{u}}(x, y, t)+\sum_{i=1}^{s} Y_{i}(t ; \omega) \mathbf{u}_{i}(x, y, t),
$$

where the $\mathbf{u}_{i}$ are orthogonal under the inner product

$$
\langle\mathbf{u}, \mathbf{w}\rangle=\int_{V} \sum_{k} u_{k} w_{k} d^{2} x,
$$

with $V$ being the flow domain and the coefficients $Y_{i}(t$; $\omega)$ are scalar stochastic processes with arbitrary probability distribution functions. The employed representation follows from the assumption that the stochastic part of the solution "lives" in a finite-dimensional space, the stochastic subspace $V_{S}$. Alternatively, one could say that the probability measure associated with the stochastic response has nonnegligible spread of probability, that is, variance, only along a finite number of dimensions.

The finite-dimensionality of the representation is crucial toward the derivation of closed field equations although it is not sufficient. The choice to have all unknown terms varying with time leads to redundancy issues and therefore additional constraints are necessary to obtain independent equations for all the quantities involved. In Sapsis and Lermusiaux (2009) it is shown that a suitable constraint, which arises naturally to overcome this redundancy issue, is the DO condition. It requires that the time-dependent basis should vary orthogonally to the space $V_{S}$ that defines on every time instant:

$$
\left\langle\frac{\partial u_{i}(\cdot, t)}{\partial t}, u_{j}(\cdot, t)\right\rangle=0, \quad i=1, \ldots, s, \quad j=1, \ldots, s .
$$

The use of time-dependence in the basis elements $\mathbf{u}_{i}(x, y, t)$ (the so-called DO modes) allows the representation of the transient character of the solution using much fewer modes. From a physical point of view what the constraint (8) imposes is the natural need for the computational algorithm to evolve the shape of the perturbations only if there is an important new direction which is not already covered by the existing modes.

The orthogonality condition leads to a closed set of equations that allows for the evolution of the mean field $\overline{\mathbf{u}}(x, y, t)$, the DO modes $\mathbf{u}_{i}(x, y, t)$, and the stochastic coefficients $Y_{i}(t ; \omega)$. This set of equations has the form of $s+1$ deterministic PDEs, defining the evolution of the mean and the DO modes, coupled to an $s$-dimensional stochastic (ordinary) differential equation that defines the evolution of the random coefficients. The DO property implies the preservation of orthonormality for the time-dependent basis $\mathbf{u}_{i}(x, y, t)$. In this way the solution is obtained in the form of random realizations for the scalar stochastic coefficients $Y_{i}(t ; \omega)$ (having low-computational and storage cost since they are low-dimensional) and in terms of $s+1$ deterministic fields containing the full deterministic information (mean field) and the $s$ timedependent directions (the DO modes) where stochasticity is important. The mean, the time-dependent patterns of the DO modes plus the distribution of the stochastic coefficients $Y$ (at a certain time $t$ ) totally specify the probability of the state vector at that time.

The expansion (6) is only exact for finite dimensional systems or for infinite dimensional systems as long as they have a finite dimensional attractor. For the case considered in this paper, the complexity of the flow is sufficiently large to capture the dynamical features that we are interested in and at the same time sufficiently small so that a small truncation dimension $s$ is adequate. The dimension $s$ can be estimated during the time development by monitoring the eigenvalues of the covariance matrix $\mathbf{C}_{Y_{i}(t) Y_{j}(t)}$ (Sapsis and Lermusiaux 2012). The criteria for evolving the subspace size are based on stability arguments which follow directly from the system differential equations. When an eigenvalue of the covariance matrix $\mathbf{C}_{Y_{i}(t) Y_{j}(t)}$ exceeds a critical value, $s$ is increased by one; when it drops below a critical value, $s$ is decreased by one. The algorithm also provides the instantaneously most unstable perturbation which is not included into the stochastic subspace.

The specific details and technicalities associated with DG equations through the DO framework can be found in the appendix; more details on the methodology can be found in Sapsis (2010). For the numerical solution of these equations a finite-volume scheme presented in Ueckermann et al. (2013) for the case of deterministic systems with random initial conditions is employed, suitably modified to take into account the stochastic part of the model.

Having the random coefficients expressed in the form of random realizations [in the simulations that follow the ensemble size is of $O\left(10^{4}\right)$ ] has important advantages especially for oceanic applications since it allows 
for the simple and efficient representation of any interesting statistical quantity of the solution such as single or joint moments of any order and probability density functions of the velocity field in specific points of the domain but also spatiotemporal correlation functions and length scales. This can be done by directly using the DO representation for fixed locations and subsequently computing sets of realizations for the velocity (or any quantity of interest) at these locations. Based on these realizations one may compute statistical moments or the associated probability density functions.

\section{Results}

In section 3 a below, we present results of the bifurcation diagram for the deterministic case with the standard values of the parameters as given in Table 1 . These results are useful as (i) they serve as a reference for the stochastic problem and (ii) they are needed for a validation of the spatial resolution used in the DO solution method. In section $3 b$, we focus on the effect of spatially coherent but temporally white noise on the statistics of DG flows and in section $3 \mathrm{c}$ we consider wind stress noise that is both temporally and spatially correlated.

\section{a. The deterministic case}

For large values of $A_{H}$, a unique and globally stable flow state for both single- and double-gyre cases is found (Dijkstra and De Ruijter 1996). To investigate the solution structure of Eq. (1) when $A_{H}$ is decreased, continuation methods (Dijkstra and Katsman 1997; Dijkstra 2005) have been used on discretized versions of (1). When the streamfunction-vorticity equations are nondimensionalized using scales $L$ for length, $U$ for velocity, $L / U$ for time, and $\tau_{0}$ for wind stress, then the dimensionless parameter $\mathrm{Re}=U L / A_{H}$ appears, which we will use as a control parameter. For the value of $A_{H}$ in Table 1, the value of $\mathrm{Re}=20$.

A $128 \times 128$ equidistant grid is used, and the steady states are computed using a pseudoarclength continuation method. For the DG wind stress forcing (3), the structure of the steady solutions is shown through the bifurcation diagram in Fig. 1a, where the value of the dimensionless streamfunction at a point in the southwest part of the domain $\left[\psi_{R}=\psi(x=L / 4, y=L / 4) /(U L)\right]$ is plotted versus $\operatorname{Re}=U L / A_{H}$.

At large values of $A_{H}$ (small Re), the antisymmetric double-gyre flow (Fig. 1b) is a unique state. When lateral friction is decreased, this flow becomes unstable at the pitchfork bifurcation $P_{1}$ and two branches (indicated by $A_{1 d}$ and $A_{1 u}$ in Fig. 1a) of stable asymmetric states appear for smaller values of $A_{H}$ (larger Re). The solutions on these branches have the jet displaced either southward
TABLE 1. Standard values of parameters used in the computations.

\begin{tabular}{cccl}
\hline \hline Parameter & \multicolumn{1}{c}{ Value } & Parameter & \multicolumn{1}{c}{ Value } \\
\hline$L$ & $1.0 \times 10^{6} \mathrm{~m}$ & $\tau_{0}$ & $1.5 \times 10^{-1} \mathrm{~Pa}$ \\
$H$ & $6.0 \times 10^{2} \mathrm{~m}$ & $\beta_{0}$ & $1.6 \times 10^{-11}(\mathrm{~m} \mathrm{~s})^{-1}$ \\
$f_{0}$ & $1.0 \times 10^{-4} \mathrm{~s}^{-1}$ & $U=\tau_{0} /\left(\beta_{0} L \rho_{0} H\right)$ & $1.56 \times 10^{-2} \mathrm{~m} \mathrm{~s}^{-1}$ \\
$\rho_{0}$ & $10^{3} \mathrm{~kg} \mathrm{~m}^{-3}$ & $A_{H}$ & $7.8 \times 10^{2} \mathrm{~m}^{2} \mathrm{~s} \mathrm{~s}^{-1}$ \\
\hline
\end{tabular}

or northward (Fig. 1c) and are exactly symmetrically related for the same value of Re. The asymmetric solutions become unstable at a Hopf bifurcation $H$ located at $\mathrm{Re}=52$.

The branch $S_{2}$ of unstable steady solutions is the continuation of the antisymmetric solution branch. For even smaller friction, this antisymmetric flow becomes inertially dominated and $\psi_{R}$ increases rapidly while going through the saddle-node bifurcation $L$. A pitchfork bifurcation $P_{2}$ occurs on the antisymmetric branch where an additional pair of asymmetric solution branches appear (indicated by $A_{2 \mathrm{~d}}$ and $A_{2 \mathrm{u}}$ in Fig. 1a); all these solutions are unstable.

The results above serve as a check for the DO method in case there is no noise in the wind stress forcing. In this case, the time evolution of the DO modes combined with their statistics through the $Y_{i}$ can be seen as a large ensemble of simulations with different initial conditions. For $\operatorname{Re}=20$, we know that all modes are damped and based on adaptive criteria (Sapsis and Lermusiaux 2012) it turns out that $s=1$ is sufficient to capture the statistics of the flow. The initial variance of the mode is chosen as $10^{-5}$ such that nonlinear interactions of the mode are initially small. The DO model is integrated for about 1 year (dimensionless time of about 0.5 ; the scaling factor is $L / U$ ) by which the evolution of the ensemble mean state, that of $10^{4}$ initial conditions and the first DO-mode is determined. As can be seen in Fig. 2a, the variance of the first DO mode (the expectation value of the square of the amplitude $Y_{1}$ ) decreases monotonically to zero and the ensemble mean flow approaches a steady double gyre flow. During the transient development, the pattern of the mean flow and the DO mode change and only the pattern at the end point of the integration $(t=0.48)$ is shown in Fig. 2a. For this case, the pattern of the DO mode, which resembles that of a Rossby-basin mode, hardly changes after $t=0.1$.

To determine the spatial resolution of the DO method and to be sure that both numerical methods give the same results for the deterministic case, we compare the equilibrium solutions of both methods for $\mathrm{Re}=20$. In Table 2, a comparison is made of the maximum and minimum values of the zonal and meridional velocity of the solution determined by the DO method and the one computed by the model in the streamfunction-vorticity 
(a)

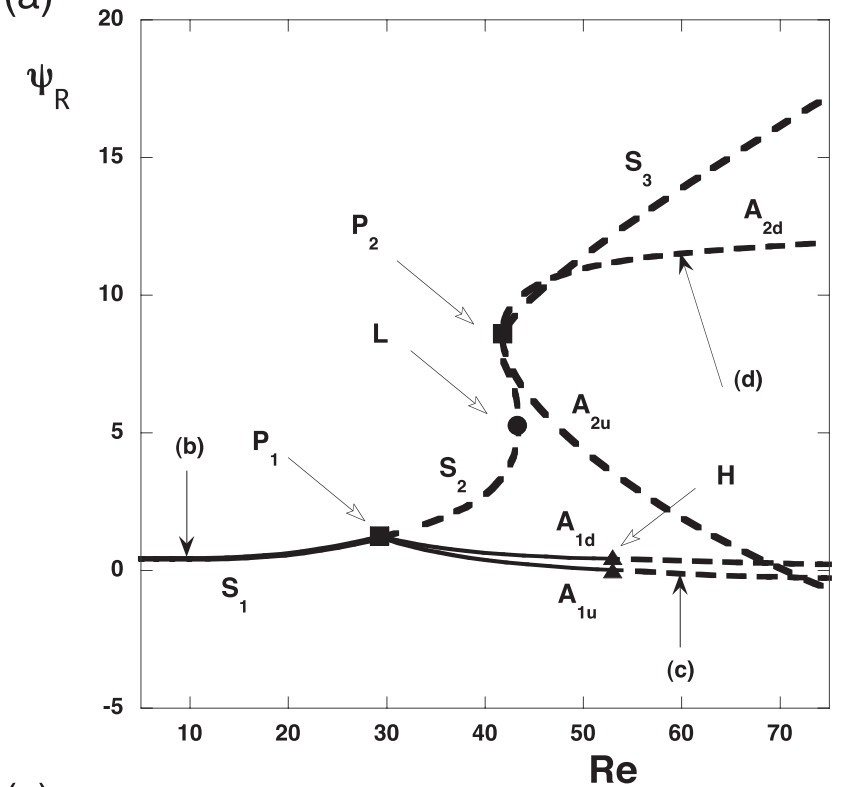

(c)

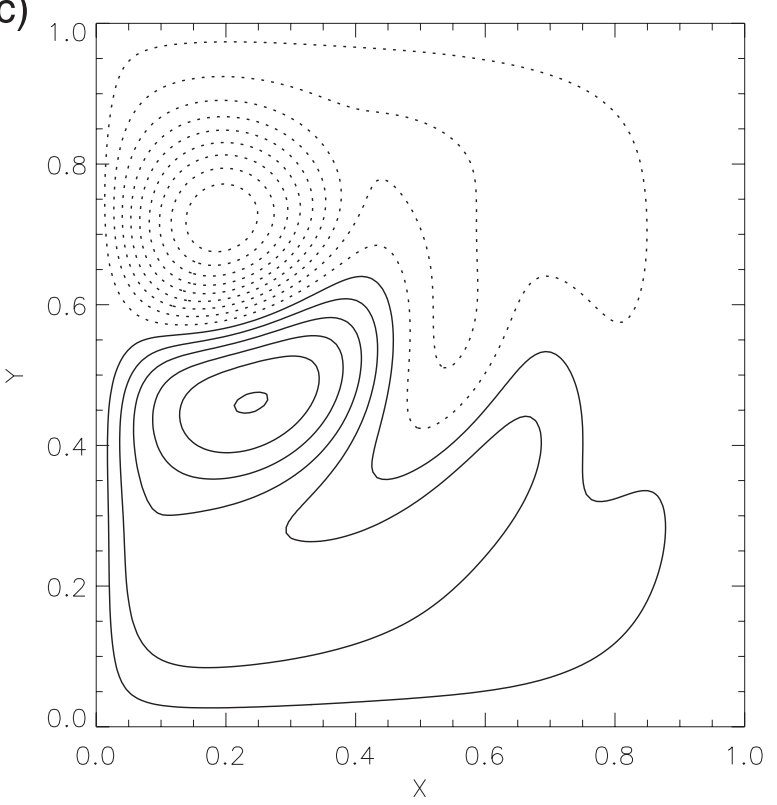

(b)

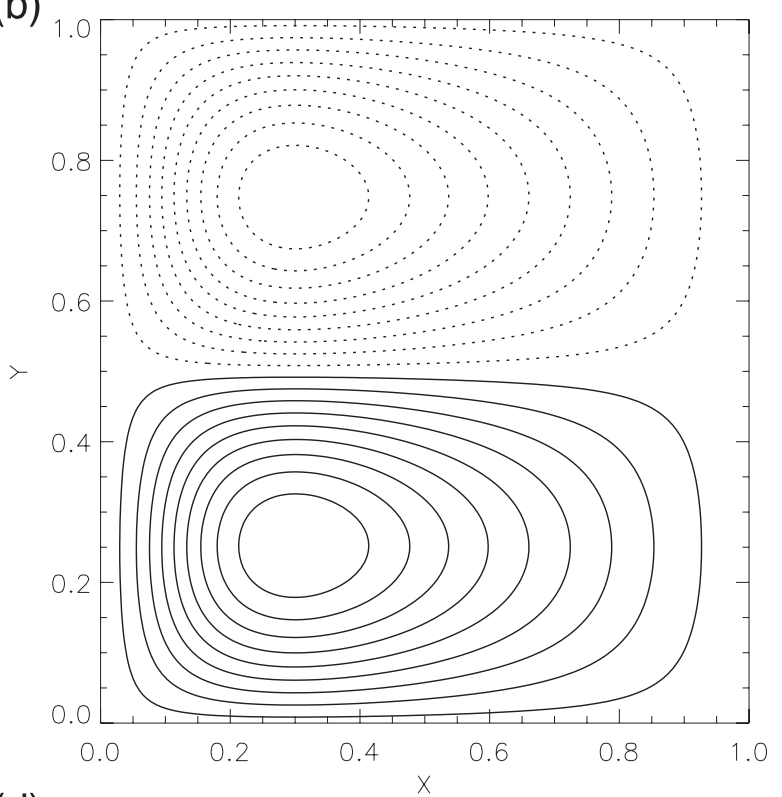

(d)

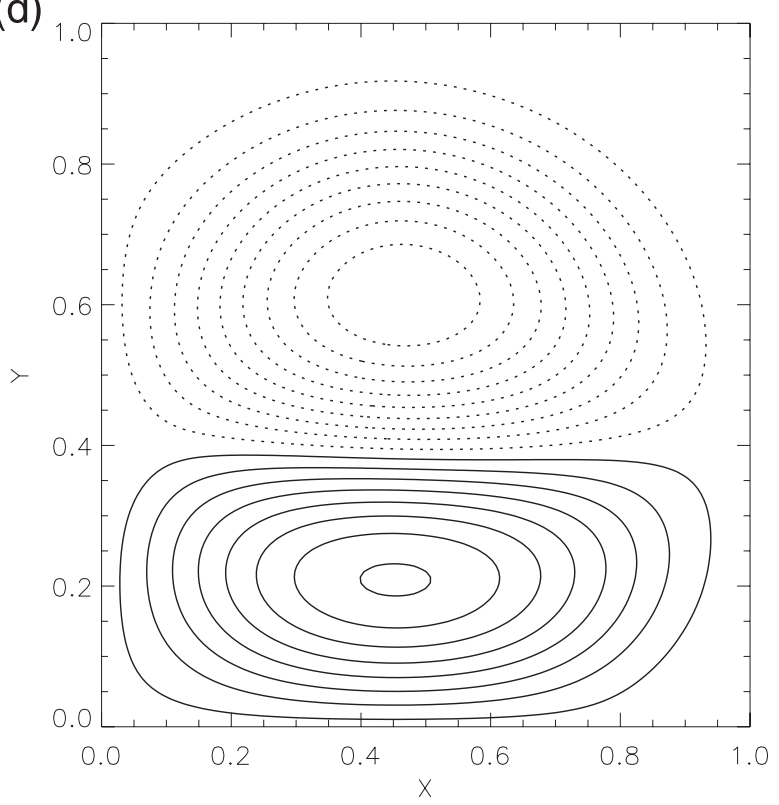

FIG. 1. (a) Bifurcation diagram for the DG barotropic quasigeostrophic model for a square basin with $\mathrm{Re}=U L / A_{H}$ as the control parameter. On the $y$ axis, the dimensionless streamfunction value $\psi_{R}=\psi(x=L / 4, y=L / 4) /(U L)$ is plotted. The Hopf bifurcation $H$ on both branches $A_{1}$ (Dijkstra and Katsman 1997) is located at $\mathrm{Re}=52$. Drawn (dashed) branches indicate linearly stable (unstable) steady states. (b) Pattern of $\psi$ near $\mathrm{Re}=10$ on the lower stable branch in (a); all contour values are with respect to the maximum value of $\psi$ and the contour spacing is 0.1 . The $x$ and $y$ coordinate are scaled with the basin size $L$. (c) Same for $\operatorname{Re}=60$ along the branch $A_{1 \mathrm{u}}$; the pattern on the branch $A_{1 \mathrm{~d}}$ at $\mathrm{Re}=60$ is the mirror image of (c) with respect to reflection through the mid axis of the basin. (d) The pattern at $\mathrm{Re}=60$ on the branch $A_{2 \mathrm{~d}}$.

formulation (1). This indicates that the DO code provides the same results as the code to solve (1) and a $64 \times 64$ grid is sufficient to obtain accurate results (an error of at most a few percent is made).

Next we consider the case $\operatorname{Re}=40$, which is in the multiple equilibrium regime (Fig. 1) with only the two asymmetric states being linearly stable. In this case, we take $s=4$ in the DO method (again based on adaptive criteria (Sapsis and Lermusiaux 2012), $s=4$ turns out to be sufficient to capture the statistics of the flow) and also with each mode having an initial variance of $10^{-5}$. The DO equations are integrated for about 12 years (up to 
a)

b)
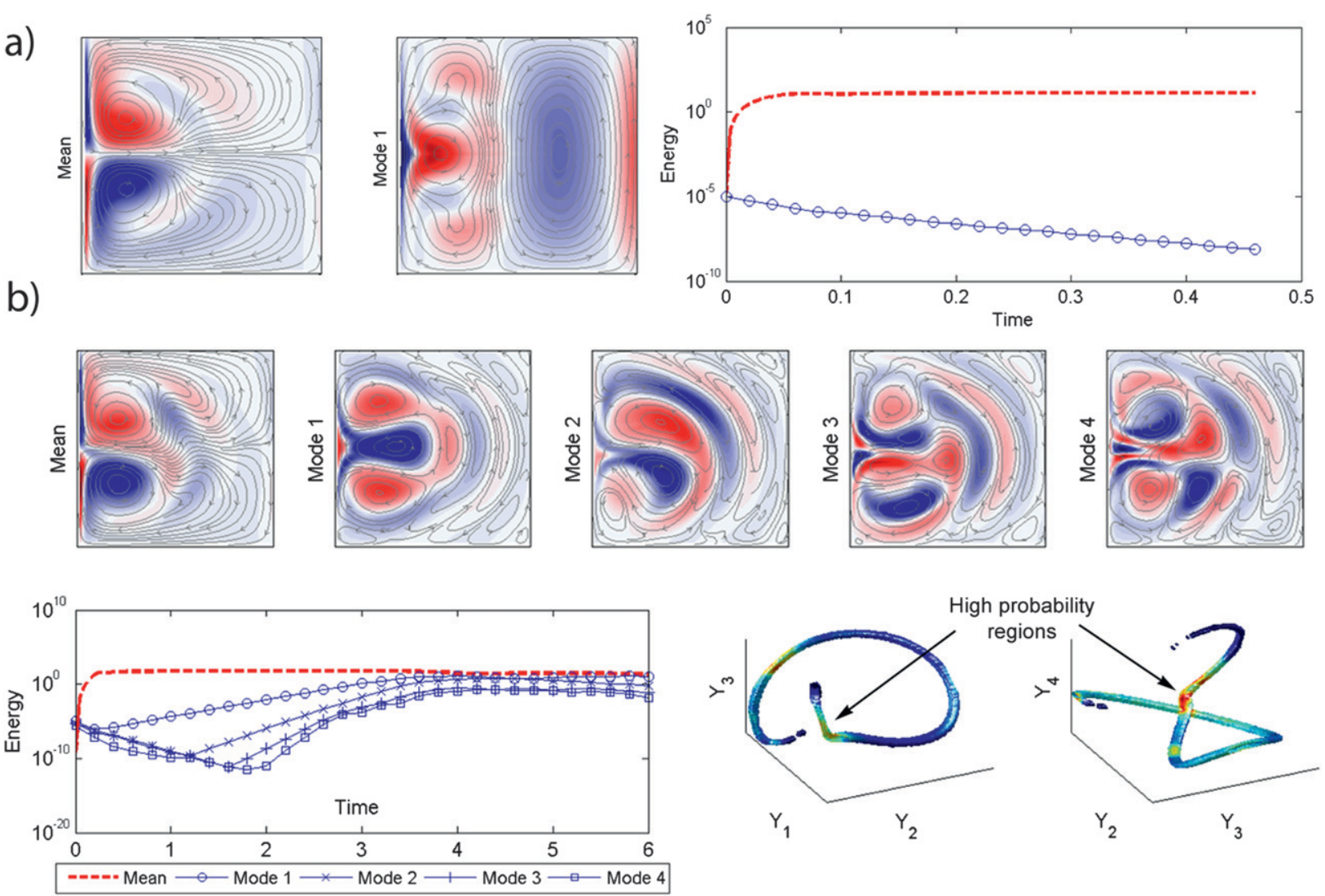

FIG. 2. (a) Solution at about one year (dimensionless time $t=0.48$ ) for the DO method for $\mathrm{Re}=20$ with $s=1$, initial variance $10^{-5}$ and a $64 \times 64$ grid (b) As in (a), but for $\operatorname{Re}=40, s=4$, and $t=6$. The color in the three-dimensional probability density functions $f_{Y_{1} Y_{2} Y_{3}}$ and $f_{Y_{2} Y_{3} Y_{4}}$ indicates its amplitude with red being high and blue being low. Since the surface corresponds to the "iso-probabilistic" location (i. e., $f_{Y_{1} Y_{2} Y_{3}}=10^{-6}$ ), the color shows the relative probability that is contained inside each location.

a dimensionless time $T=6$ ) and the results are shown in Fig. 2b. The streamfunction (contours) and vorticity (color) of the mean and DO modes are shown in the upper panels, the time development of the DO modes in the lower-left panel and two three-dimensional representations of the four-dimensional PDF of the system are presented in the lower-right panel. In the latter panel, regions with high probability are shown in red. As can be seen in Fig. 2b, the amplitudes of the DO modes approach finite amplitude values. The pattern of DO mode 1 is similar to that of the so-called $\mathrm{P}$ mode (Simonnet and Dijkstra 2002), which is the pattern of the eigenmode that destabilizes the symmetric steady state at the pitchfork bifurcation $P_{1}$ (Fig. 1b) through symmetry breaking. The pattern of DO mode 2 is the socalled L mode (Simonnet and Dijkstra 2002), which is here slightly asymmetric as the mean state is asymmetric. The patterns of DO modes 3 and 4 are similar to DO modes 1 and 2, but with higher zonal wavenumbers.

From the results in Fig. 2 we deduce that different statistical regimes can be distinguished by looking at the decay of the DO modes. In Fig. 3b, the logarithm of the amplitude of the leading DO mode is plotted as a function of time for different values of Re. This shows that for values $\operatorname{Re}<30$, the amplitudes of the DO modes decay whereas some of them do not decay for $\operatorname{Re}>30$. This result is in correspondence with the occurrence of the pitchfork bifurcation $P_{1}$ in Fig. 1 . Note that the patterns of the mean and the DO modes change with

TABLE 2. Comparison of dimensionless maximum and minimum values of zonal and meridional velocities of the steady state in both streamfunction-vorticity (indicated by QG below) and velocitypressure (DO) models for $\mathrm{Re}=20$ and different spatial resolutions.

\begin{tabular}{lccc}
\hline \hline Resolution & $u_{\min }$ & $u_{\max }$ & $v_{\max }=-v_{\min }$ \\
\hline DO $64 \times 64$ & -4.2360 & 14.7943 & 11.6493 \\
QG $64 \times 64$ & -4.2669 & 14.4148 & 11.6233 \\
DO $96 \times 96$ & -4.4387 & 14.9021 & 11.8784 \\
QG $96 \times 96$ & -4.4014 & 14.8310 & 11.7839 \\
DO $128 \times 128$ & -4.4351 & 14.9342 & 11.8945 \\
QG $128 \times 128$ & -4.4646 & 14.9720 & 11.8997 \\
\hline
\end{tabular}



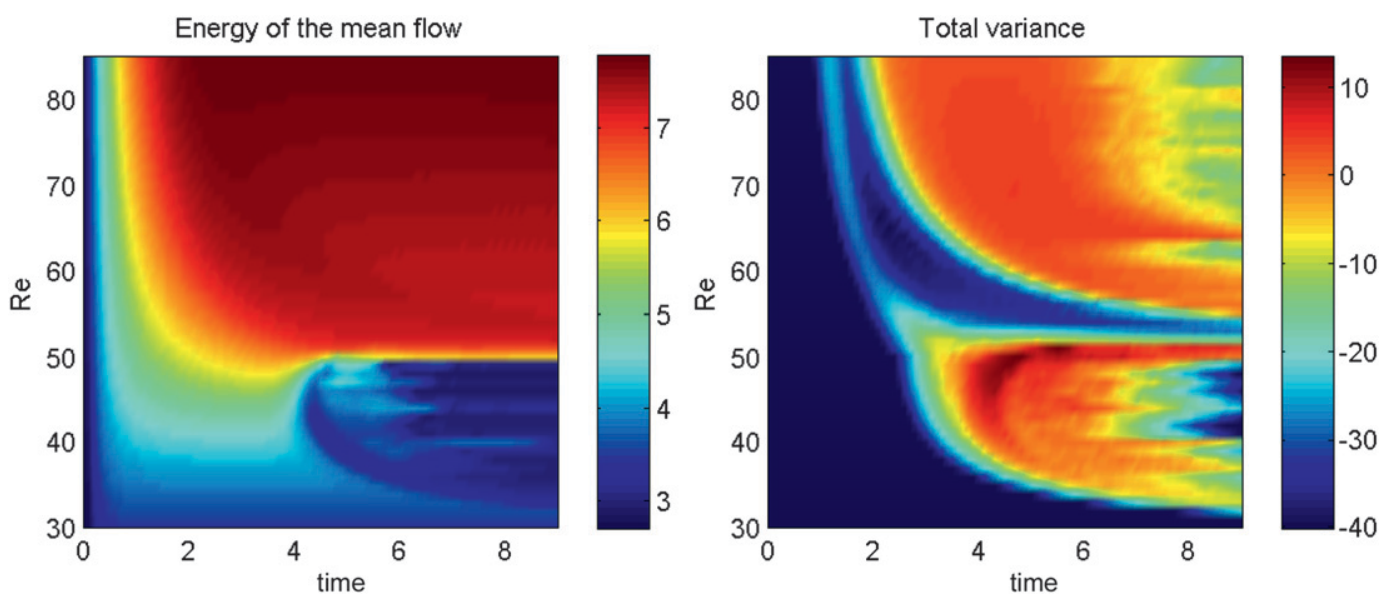

FIG. 3. Logarithm of (a) the energy of the mean and logarithm of (b) total variance of the leading DO mode, both vs time for different values of Re.

time and are not yet equilibrated for $t=6$ (for which their pattern is shown in Fig. 2b). The logarithm of the energy of the mean is plotted in Fig. 3a and for $30<$ $\operatorname{Re}<50$ it gradually increases. Figure 1 indicates that there are only two stable asymmetric states in this Re regime and hence the time mean is expected to be the antisymmetric state, which has a nonzero mean energy and increases in amplitude with Re. The energy of the mean undergoes a rapid increase for values of $\operatorname{Re}>50$. This is associated with the occurrence of the Hopf bifurcation $H$ on the branches of asymmetric solutions (Fig. 1a) and the subsequent rectification of the mean flow through the periodic solutions arising from this bifurcation (Dijkstra and Katsman 1997). There are also two regimes of variance of the leading DO mode (Fig. 3b), one associated with the multiple equilibrium regime $(\operatorname{Re}<50)$ and one with an essentially transient regime $(\operatorname{Re}>50)$. In the analysis of the effects of noise, we focus only (as also did Sura et al. 2001 and Pierini 2010) on the first (multiple equilibrium) regime.

\section{b. Additive white noise wind stress forcing}

For the stochastic wind stress component $\tau^{\prime}$ we follow Sura et al. (2001) and prescribe it as

$$
\tau^{\prime}=\rho_{a} C_{D}\left|\mathbf{u}^{\prime}\right| \mathbf{u}^{\prime}
$$

with

$$
\begin{gathered}
u^{\prime}=\sigma \eta_{x}(t) f(x, y) ; \quad v^{\prime}=\sigma \eta_{y}(t) f(x, y) \\
f(x, y ; \lambda)=\frac{\alpha}{\sqrt{\pi}}\left[\lambda \operatorname{Erf}\left(\frac{1}{2 \lambda}\right)\right]^{-1} e^{-\frac{\left(\frac{x}{L}-\frac{1}{2}\right)^{2}+\left(\frac{y}{L}-\frac{1}{2}\right)^{2}}{2 \lambda^{2}}}
\end{gathered}
$$

where $\lambda=L_{x} / L=L_{y} / L$ is a scale factor controlling the spatial extent ( $L_{x}=L_{y}$ in Sura et al. 2001) of the stochastic noise and $\alpha$ controls the amplitude. In this subsection, the quantities $\eta_{x}$ and $\eta_{y}$ represent uncorrelated (white) noise with a unit intensity.

Similarly to the choice in Sura et al. (2001), the amplitude $\alpha$ is normalized so that it is the same for every value of $\lambda$, that is, $\alpha$ is computed from $\alpha=1 / f(0,0 ; 1 / 4)$. The reference amplitude for $\lambda=1 / 4$ is chosen such that the variance in the noise $\sigma^{2}=28 \mathrm{~m}^{2} \mathrm{~s}^{-2}$, similar to that in Sura et al. (2001), with also values of $\rho_{a}=1.2 \mathrm{~kg} \mathrm{~m}^{-3}$ and $C_{D}=2.0 \times 10^{-3}$. In this case, we have only two control parameters to which we want to investigate the sensitivity of the response, that is, $\lambda$ and Re.

The DO results for $\mathrm{Re}=35$ are shown in Fig. 4 for three values of $\lambda$ in the same format as Fig. 2b. For each value of $\lambda$, the amplitudes of all DO modes approach steady values in time and the first DO mode (of which the pattern corresponds to the normal mode associated with the symmetry breaking at the bifurcation point $P_{1}$ in Fig. 1) has the largest amplitude. The remarkable result here is that for each of the three values of $\lambda$, we find that the spatial structure of the DO modes is the same. Also the geometry of the probability density function the same for different noise forcing although the probability measure is more spread for smaller values of $\lambda$.

To understand this behavior, we first reformulate corollary 2 from Sapsis and Lermusiaux (2009) to the following form, most appropriate for this context. Consider the stochastic partial differential equation

$$
\begin{gathered}
d u_{t}(x, t ; \omega)=\mathcal{L}[u(x, t ; \omega)] d t+\sum_{k=1}^{s} d W_{k}(t ; \omega) \sigma_{k}(x, t), \\
x \in V, \quad t \in T, \quad \omega \in \Omega
\end{gathered}
$$


a)
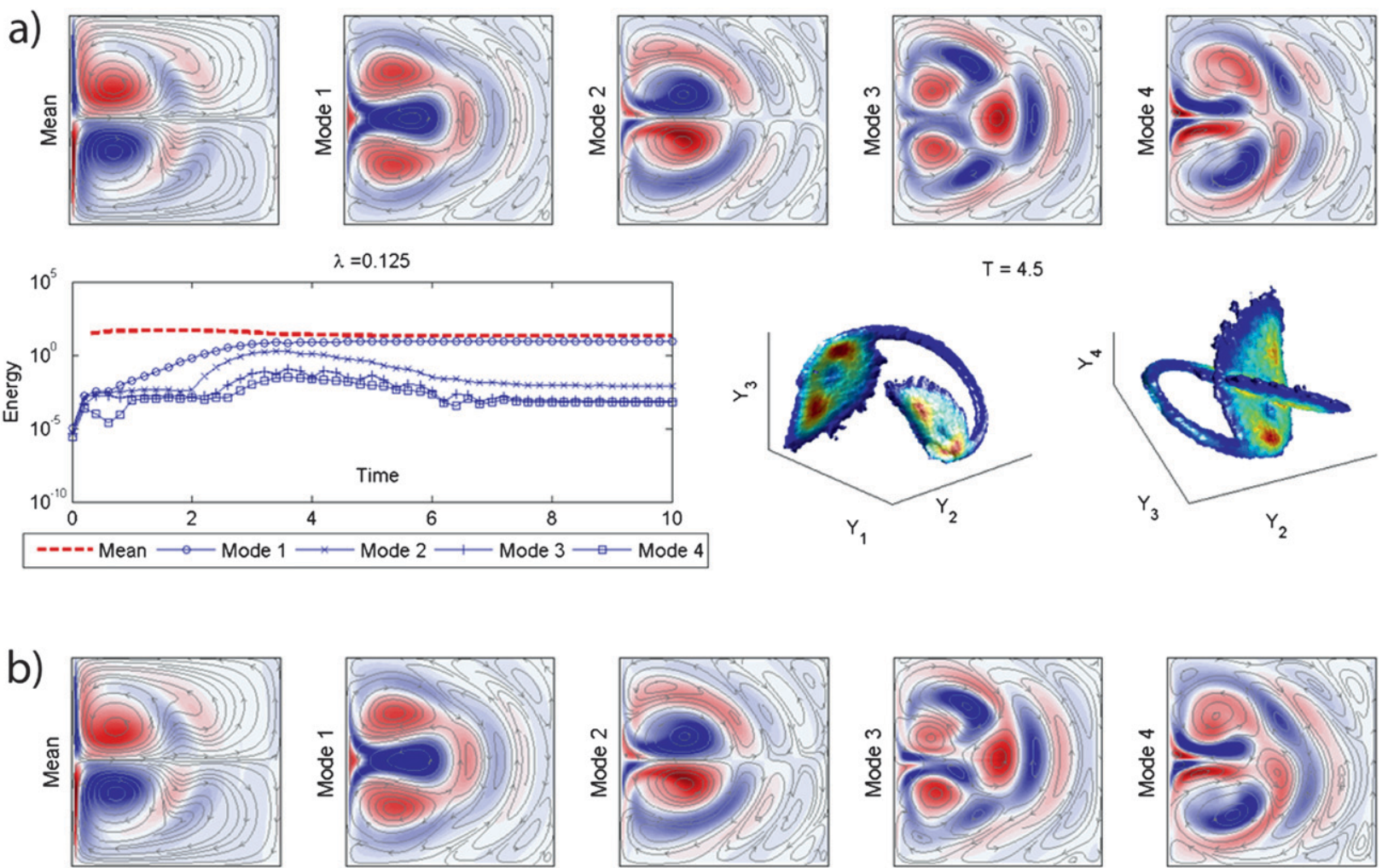

$\mathrm{T}=4.5$
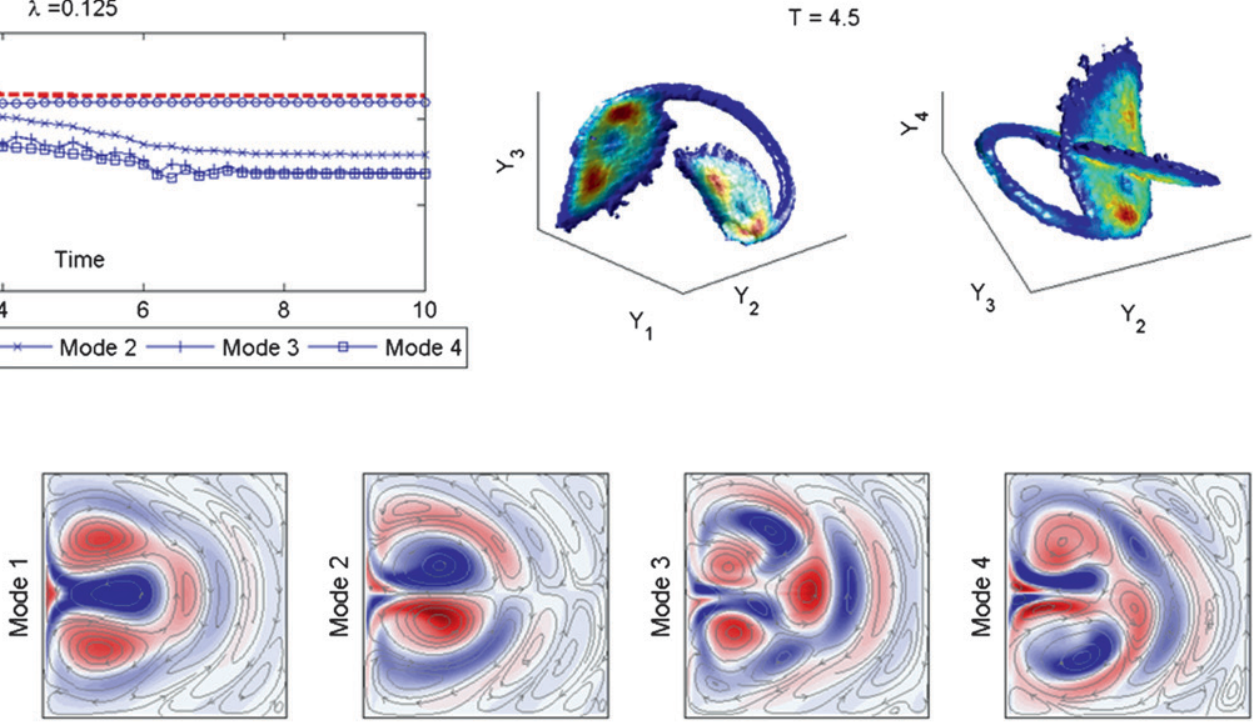

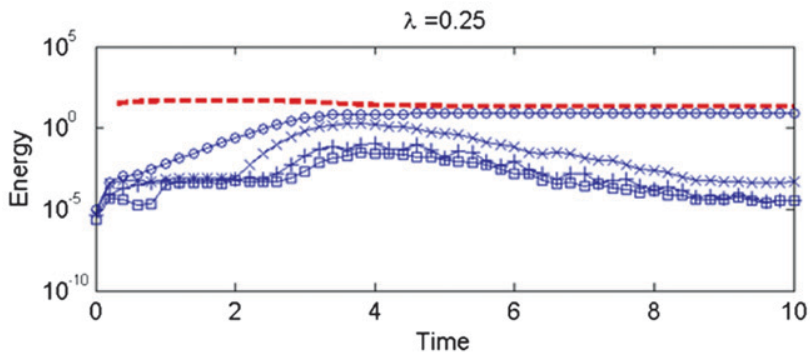

$\mathrm{T}=4.5$
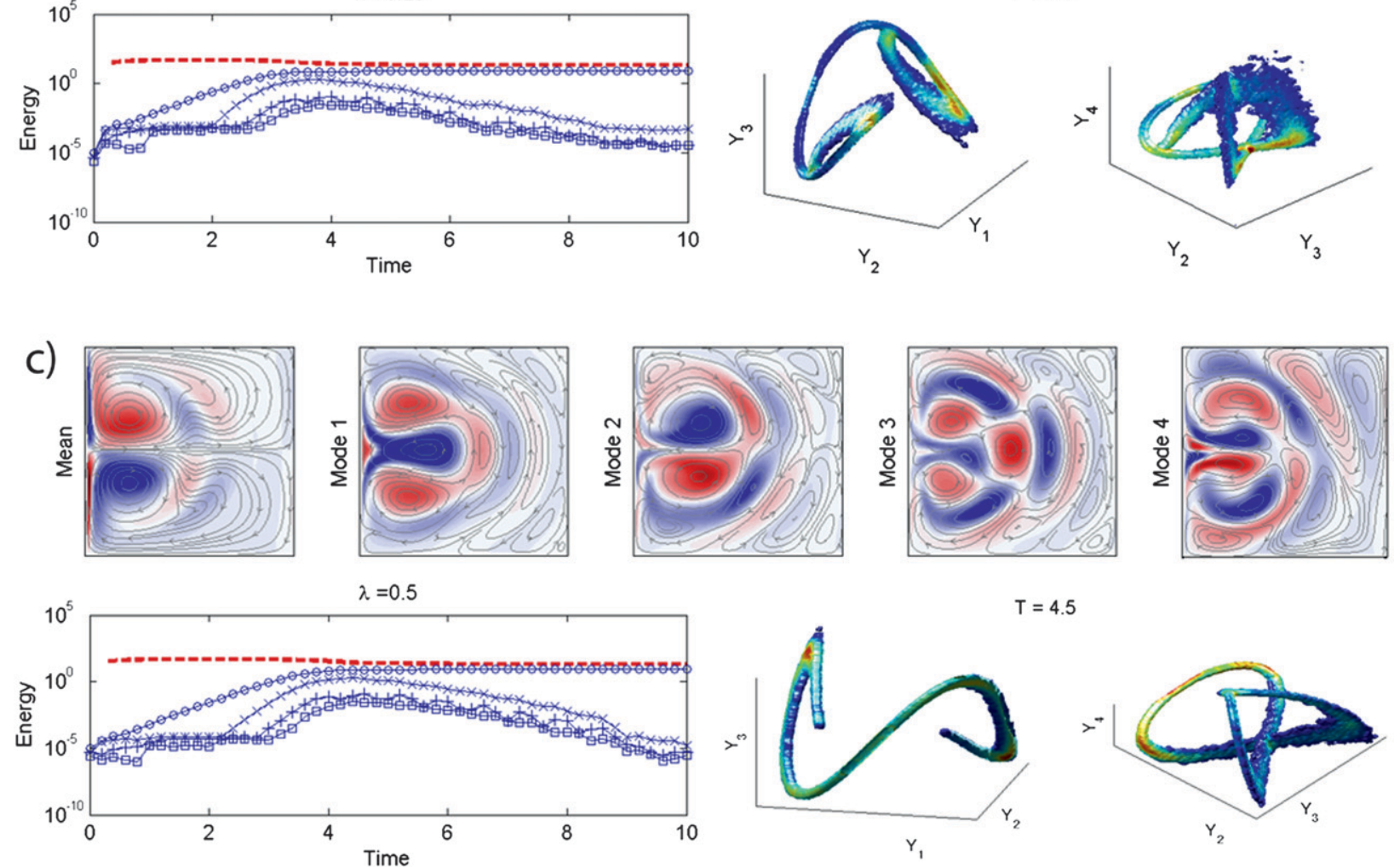

FIG. 4. DO results in time for $\mathrm{Re}=35$ as determined on a $64 \times 64$ grid, with $\mathrm{s}=4$, initial variance $10^{-5}$, a scaled noise amplitude $\alpha$, and three values of (a) $\lambda=0.125$, (b) $\lambda=0.25$, and (c) $\lambda=0.5$. The total time integrated is about 20 years $(T=10)$. 
where $\mathcal{L}$ is a general (nonlinear), differential operator, and $W_{k}(t ; \omega)$ are independent Wiener processes. Then the stochastic excitation has zero effect on the instantaneous evolution of the mean field, and the shape of the stochastic subspace $V_{S}$, while for the stochastic coefficients we have the following two cases.

(i) If $\sigma_{k}(x, t) \in V_{S}$ then the effect of the excitation is expressed as diffusion in the reduced order stochastic subspace and only on the stochastic coefficients $Y_{i}(t ; \omega)$ for which we have $\left\langle\sigma_{k}(x, t), u_{i}(x, t)\right\rangle \neq 0$ for some $k=1, \ldots, s_{e}$.

(ii) The forcing components for which $\sigma_{k}(x, t) \notin V_{S}$ have zero effect on the instantaneous evolution of the DO solution.

According to the results in Fig. 4, we expect that white noise excitation will not cause significant changes on the form of the reduced-order space since its effect can only be indirect to it (through the influence on the stochastic coefficients). To give a more quantitative characterization of the effect of white-noise on the system dynamics we compute the rate of energy transfer and dissipation involving each DO mode and the mean. We will use $\varepsilon_{\mathrm{diss}, i}$ to indicate the dissipation of energy by DO mode $i, \varepsilon_{\text {mean } \rightarrow i}$ the energy transfer from the mean to DO mode $i, \varepsilon_{\mathrm{DO} \rightarrow i}$ the exchange of energy from all other DO modes to DO mode $i$ and $\varepsilon_{\mathrm{ext}, i}$ the exchange of energy of the stochastic forcing to DO mode $i$. When the wind stress forcing is written in general as (see appendix)

$$
\tau^{\prime}(\mathbf{x}, t)=\sum_{k=1}^{s} Z_{k}(t ; \omega) \sigma_{k}(\mathbf{x}, t)
$$

the energy transfer rates can be expressed analytically in terms of the statistics of the system as (see Sapsis 2010, section 5.5 or Sapsis 2012, manuscript submitted to Proc. Roy. Soc.)

$$
\begin{aligned}
\varepsilon_{\mathrm{diss}, i} & =-E^{\omega}\left[Y_{i}^{2}\right] \int_{V} \frac{1}{\operatorname{Re}} \nabla \mathbf{u}_{i}, \nabla \mathbf{u}_{i} d \mathbf{x}, \\
\varepsilon_{\text {mean } \rightarrow i} & =-E^{\omega}\left[Y_{i}^{2}\right] \int_{V} \mathbf{u}_{i}^{\mathrm{T}} \mathbf{S}_{\overline{\mathbf{u}}} \mathbf{u}_{i} d \mathbf{x}, \\
\varepsilon_{\mathrm{DO} \rightarrow i} & =-\int_{V} \mathbf{u}_{q}^{\mathrm{T}} \mathbf{S}_{\mathbf{u}_{i}} \mathbf{u}_{p} d \mathbf{x} E^{\omega}\left[Y_{i} Y_{p} Y_{q}\right], \\
\varepsilon_{\mathrm{ext}, i} & =\mathbf{C}_{Z_{k}(t) Y_{i}(t)} \int_{V} \sigma_{k}(\mathbf{x}, t) \cdot \mathbf{u}_{i}(\mathbf{x}, t) d \mathbf{x}
\end{aligned}
$$

where in the integrals above, the Einstein summation convention is applied. In addition, $E^{\omega}$ indicates the expectation value, $\mathbf{C}$ the covariance matrix and

$$
\left(S_{v}\right)_{n m}=\frac{1}{2}\left(\frac{\partial v_{n}}{\partial x_{m}}+\frac{\partial v_{m}}{\partial x_{n}}\right) .
$$

We consider the following three cases: the system without any external stochastic noise, and the system under white stochastic excitation in the wind stress with two different length scales $\lambda$ of the spatial structure of the noise. We emphasize that due to the nonanticipative property of white-noise the energy transferred from the stochastic excitation to the DO modes is zero. In particular, one can prove from Eq. (A1) in the appendix that the energy transfer rate from the external stochastic forcing to each DO mode for white-noise $\varepsilon_{\mathrm{ext}, i}=0$ since we always have $\mathbf{C}_{W_{k}(t) Y_{i}(t)}=0$.

By plotting the above energy transfer rates in Fig. 5 we observe that the response of the system in these three cases is almost identical in terms of energy transfer properties. Because the white noise in the forcing is uncorrelated with the amplitudes of the DO modes, there is only a relative shift of the time series, while the overall energetics remains the same. In particular the deterministic system is the one where the instability takes longer to develop while the system subjected to noise with the smaller length scale (or noise with sharper spatial characteristics) is the one that develops substantial uncertainty the earliest.

In addition, in all three cases considered in Fig. 5, we notice that there are two (linearly unstable) DO modes that absorb energy from the mean flow (DO modes 1 and 2). However, these two modes behave completely differently if we take into account the nonlinear energy transfer properties. In particular, DO mode 1 absorbs energy not only from the mean flow but also from the other DO modes through nonlinear interactions. These two kinds of energy fluxes (linear and nonlinear) are balanced by the strong rate of energy dissipation. On the other hand, DO mode 2 transfers an important amount of its energy to DO modes 3 and 4 which dissipate some of it and return the rest to the mean flow. In all cases we observe that the presence of white-noise does not disturb this energy cycle.

To emphasize the similarity between the different excitation scenarios we present in Fig. 6 the response for each one with an appropriate time shift relative to the deterministic case which is chosen in accordance with the time delay of the energy time series. Consistently with the general formulation, the stochastic subspace and the mean remain almost unchanged in all three cases of excitation albeit the time delay. In Fig. 6 we also present a visualization of the probability density function through the contours $\left\{\left(Y_{1}, Y_{2}, Y_{3}\right) \mid f_{Y_{1} Y_{2} Y_{3}}=10^{-6}\right\}$ and $\left\{\left(Y_{2}, Y_{3}, Y_{4}\right) \mid f_{Y_{2} Y_{3} Y_{4}}=10^{-6}\right\}$ for the three cases. Because of the low value that these contours correspond to, most of the probability mass is contained inside these closed surfaces. Moreover, the coloring of the surfaces is according to the contained probability at 

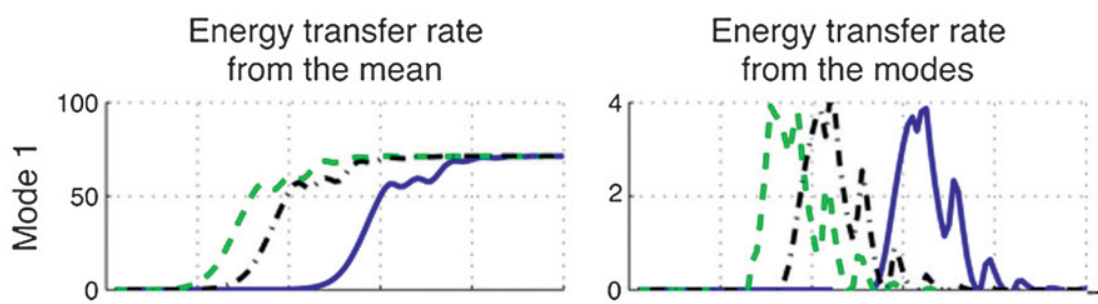

\section{Energy dissipation rate}
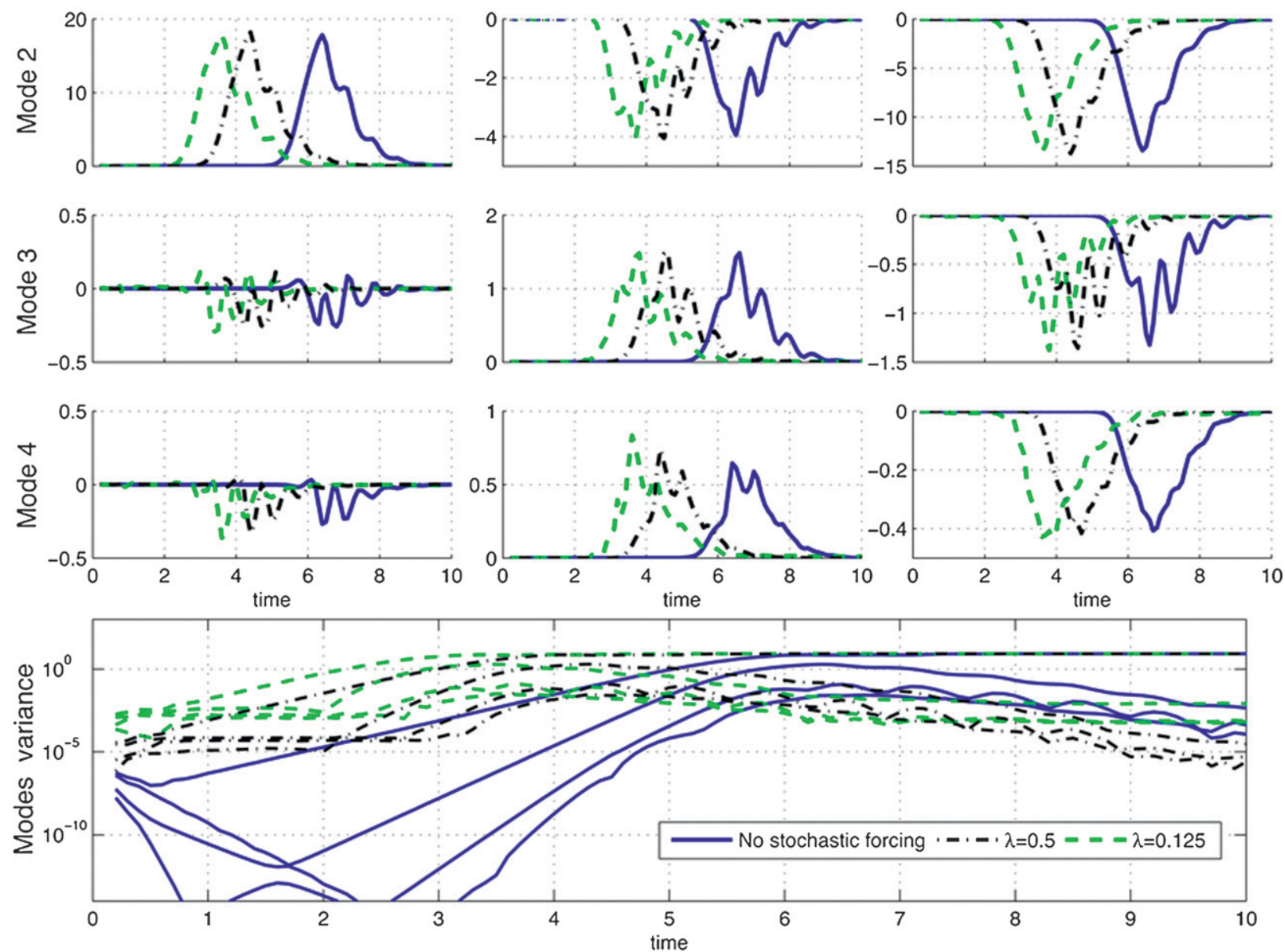

FIG. 5. Rates of energy transfer to each mode for the case of deterministic excitation (blue solid curve) and in the presence of white noise excitation for two different spatial correlation length scales $\lambda$.

each location of the contour (red regions corresponding to high probability and blue to low values).

For both the deterministic and the stochastic cases we see that the probability density function essentially consists of two different kinds of regions: (i) locations where the probability is spreading along a 1-dimensional manifold and, (ii) regions where the probability density function "lives" in two-dimensional sets. As discussed in detail in Sapsis (2012, manuscript submitted to Proc. Roy. Soc.) these two different regions indicate locations of the reduced-order subspace where a different number of linear instabilities occur. In particular in regions where we have a one-dimensional probability density function there is only one linearly unstable mode (in this case this is Mode 1) while in locations where we have a twodimensional probability density function there are two linear instabilities coexisting. The presented results are in full consistency with the energy transfer properties presented and discussed previously.

\section{c. The case of temporally correlated stochastic excitation}

We now consider the most general case where the wind stress noise has a correlated-in-time component to 

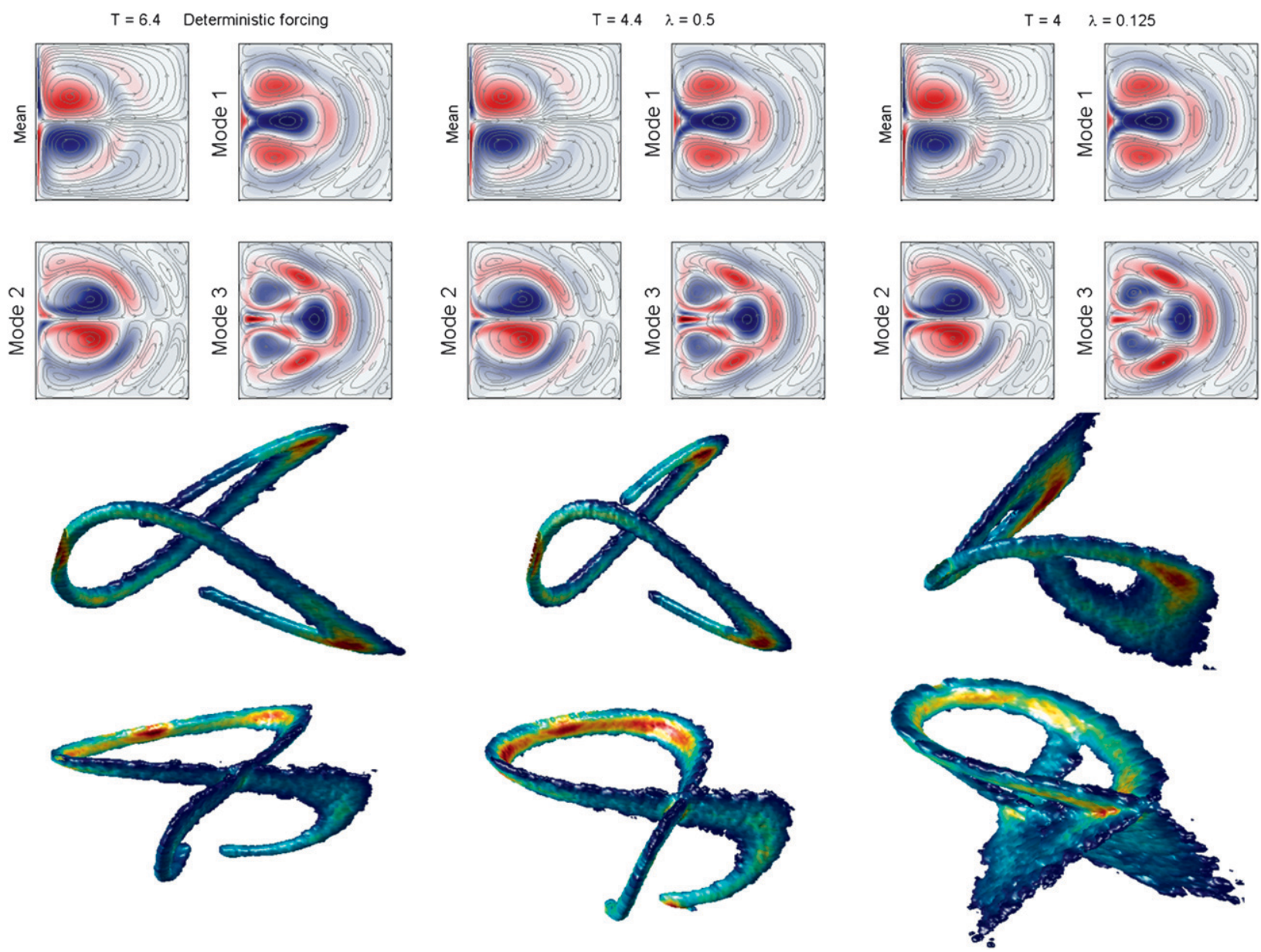

FIG. 6. Mean flow and stochastic subspace in terms of the streamfunction (contours) and vorticity field (colors) as well as contour plots of the three-dimensional marginals $f_{Y_{1} Y_{2} Y_{3}}$ and $f_{Y_{2} Y_{3} Y_{4}}$ for the case of deterministic forcing and for two cases of white-noise stochastic forcing with two different spatial characteristics. The three cases have been plotted with appropriate time delay (obtained by the energy curves) so that we have correspondence to the same dynamical regime.

determine the effect of memory in the stochastic excitation on the statistics of the DG flows. In particular we assume that the stochastic processes $\eta_{x}(t ; \omega)$ and $\eta_{y}(t ; \omega)$ in (10) are solutions of the following stochastic differential equations

$$
\begin{aligned}
& d \eta_{x}(t ; \omega)=-\frac{1}{\tau} \eta_{x}(t ; \omega) d t+\sqrt{\frac{2}{\tau}} d W_{x}(t ; \omega) \\
& d \eta_{y}(t ; \omega)=-\frac{1}{\tau} \eta_{y}(t ; \omega) d t+\sqrt{\frac{2}{\tau}} d W_{y}(t ; \omega) .
\end{aligned}
$$

The solution of each of the SDEs (11) are zero mean Gaussians (Gitterman 2005; Henderson and Plaschko 2006) with covariance function $C(t)=e^{-t / \tau}$. Therefore each of those stochastic processes will correspond to a decorrelation time scale $\tau$ and variance $E^{\omega}\left[\eta_{x}^{2}(t ; \omega)\right]=$ $E^{\omega}\left[\eta_{y}^{2}(t ; \omega)\right]=1$. We emphasize that as $\tau \rightarrow 0$, each of the processes $\eta$ converges to a white noise process with a $\sqrt{2 \tau}$ intensity (Gardiner 2002). Therefore for the limit of $\tau=0$, we should recover the deterministic dynamics. However, as we will see below, this is not the case and a singular limit behavior occurs. Note that the particular choice of model for each $\eta$ (having constant variance) is made so that we examine exclusively the effect of memory in the excitation process and not the effect of its magnitude.

In Fig. 7 we present the energy transfer rates for three cases of decorrelation time scale $\tau$ as in (18) with fixed $\lambda=1 / 8$. We observe that as the decorrelation time (or memory) exceeds a certain limit the system behaves completely different from the deterministic or the white noise excitation (effectively $\tau=0$ ) and it is characterized by much stronger energy transfer rates and energy content. This is also the case for the DO modes which characterize the reduced order stochastic subspace, shown in Fig. 8. 

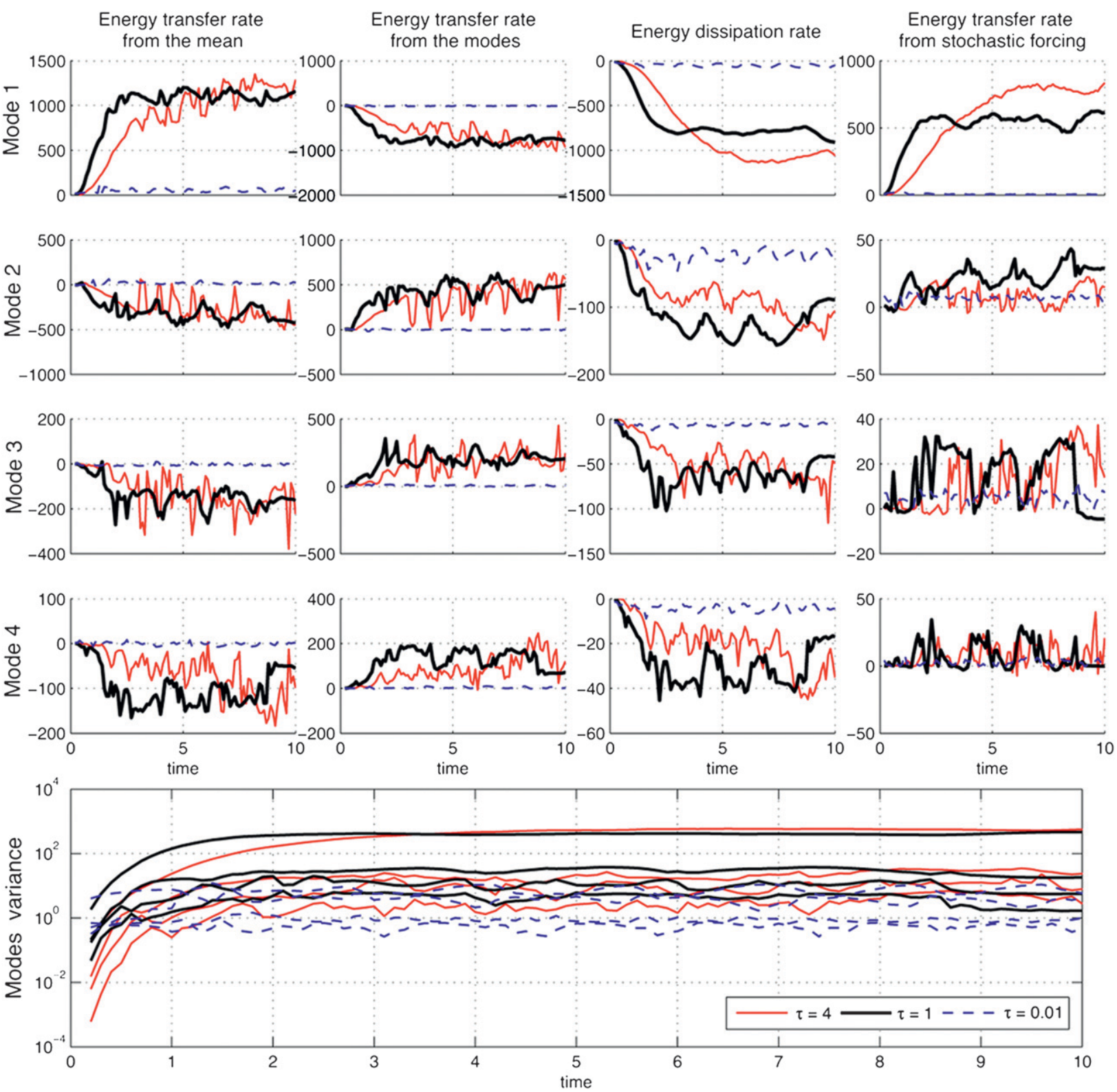

FIG. 7. Energy transfer rates for the cases of correlated in time stochastic excitation and for three different decorrelation time scales $\tau$. For all cases the spatial parameter $\lambda$ is fixed and equal to $1 / 8$ while the decorrelation time scale $\tau$ is taken 0.01 (blue), 1 (black) and 4 (red).

As the decorrelation time scale $\tau$ increases, essentially the excitation behaves more deterministically (longer memory) and the system enters into a completely different dynamical regime characterized by a different set of modes but most importantly different energy transfer properties (Fig. 7). In contrary to the deterministic or white-noise excitation cases, here we have only a single linearly unstable mode (mode 1 ). In the white-noise case this mode was also absorbing energy from the other DO modes. The presence of longmemory excitation changes this behavior and now this mode transfers energy to all the other DO modes which dissipate some and return the rest to the mean flow. Note that in addition to the internal energy transfers here we have all the stochastic modes to absorb energy directly from the random excitation. The above facts are also consistent with the geometry of the probability density function (Fig. 8) that has a one-dimensional structure with a small two-dimensional component (which corresponds to a dynamical regime where we have two DO modes absorbing energy from the mean flow). 


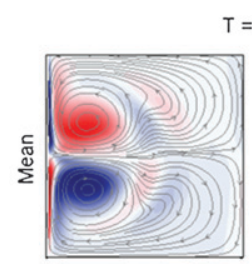

$\mathrm{T}=7 \quad \tau=0.01$
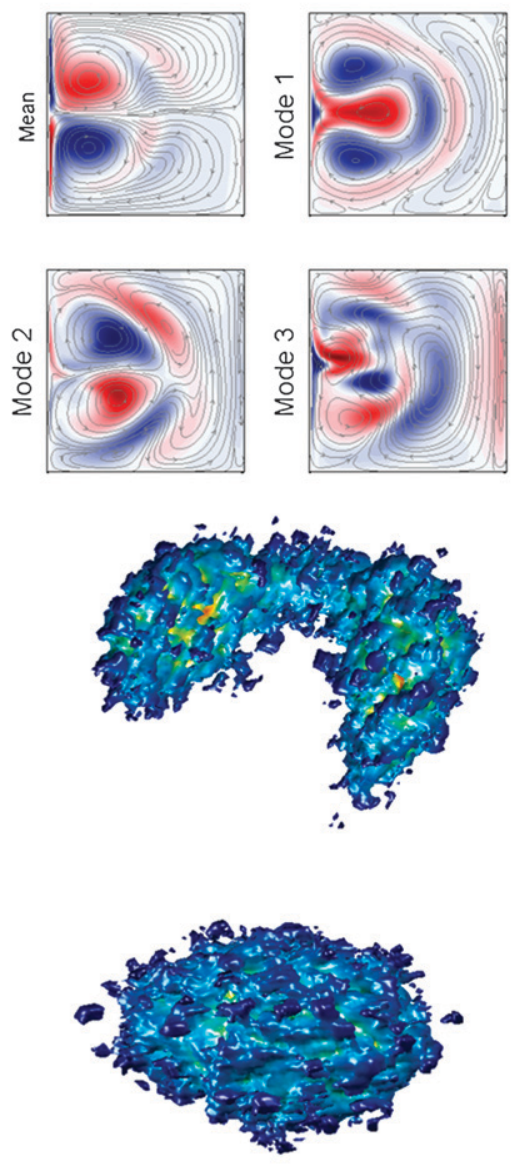

$\mathrm{T}=7 \quad \tau=1$
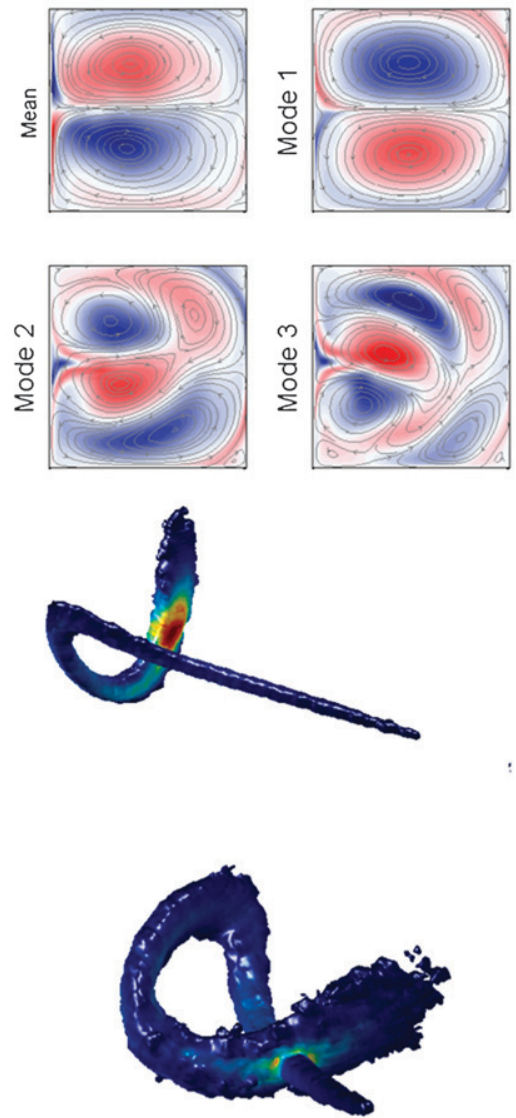

$\mathrm{T}=7 \quad \tau=4$
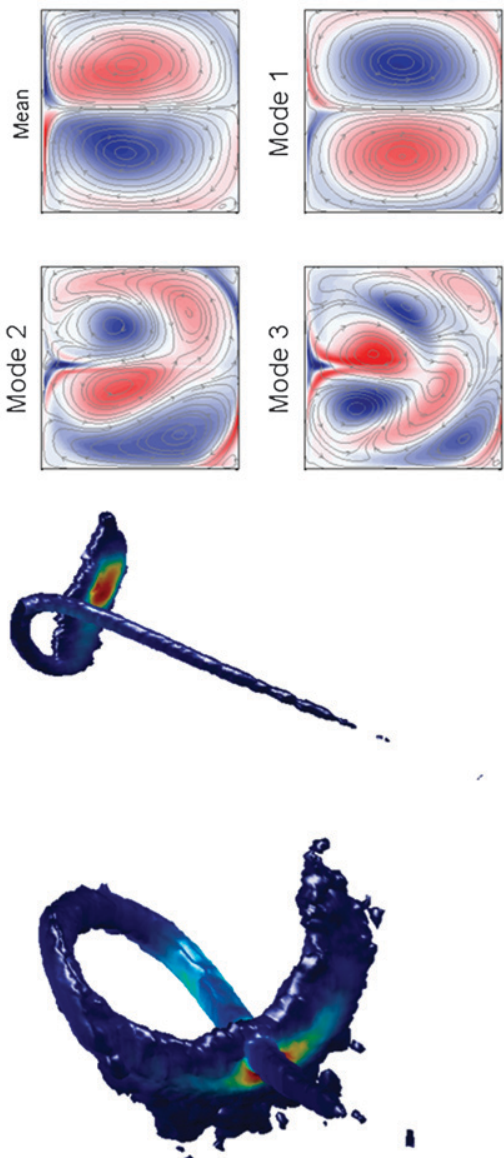

FIG. 8. Mean flow, DO modes 1-3 and probability density functions for the case of temporally and spatially correlated noise excitation for $\lambda=1 / 8$ and three different decorrelation time scales: $\tau=0.01,1$, and 4 .

The case $\tau=0.01$ is considered in more detail in Fig. 9. We see that for the small decorrelation time scale the energy transfer rates are closer in magnitude to that of the white-noise excitation. However, the energy transfer properties are completely different from those of the deterministic or white-noise excitation cases as for small $\tau$ the amplitudes do not become stationary but keep fluctuating. We see that DO modes 1 and 2 remain linearly unstable but with amplitudes that fluctuate in time in a statistically stationary faction. Moreover, DO modes 3 and 4 lose their robustly stable character and in many time instants they absorb energy directly from the mean flow. However, the major role on importing energy to them is played by their direct interaction with the stochastic excitation but also the energy in-flux through nonlinear interactions with the other DO modes. These differences are also expressed in the probability density functions (Fig. 8 , for $\tau=0.01$ ) where we see that the dimensionality of their support is larger than two (which was the dimensionality in the case of deterministic excitation) a fact that is connected to the number of unstable modes as we discussed previously. The limit $\tau \rightarrow 0$ appears to be a singular limit and its discussion is outside the scope of this paper.

In summary, we have shown that stochastic noise characterized by finite-memory acts drastically on the reduced order dynamics of the system causing changes in the stability of the flow. Depending on the memory of the excitation, this may destabilize modes of the deterministic system pushing the dynamics into a statistically stationary regime (small memory), or bring the system into a less complex behavior characterized only by a single unstable mode (long memory).

\section{Summary and discussion}

Using the dynamically orthogonal (DO) field method, the effect of additive noise on the double-gyre winddriven flows was studied using an idealized quasigeostrophic model. In the DO method the solution of the stochastic barotropic vorticity equation is found by a Karhoenen-Loeve expansion into so-called DO modes 

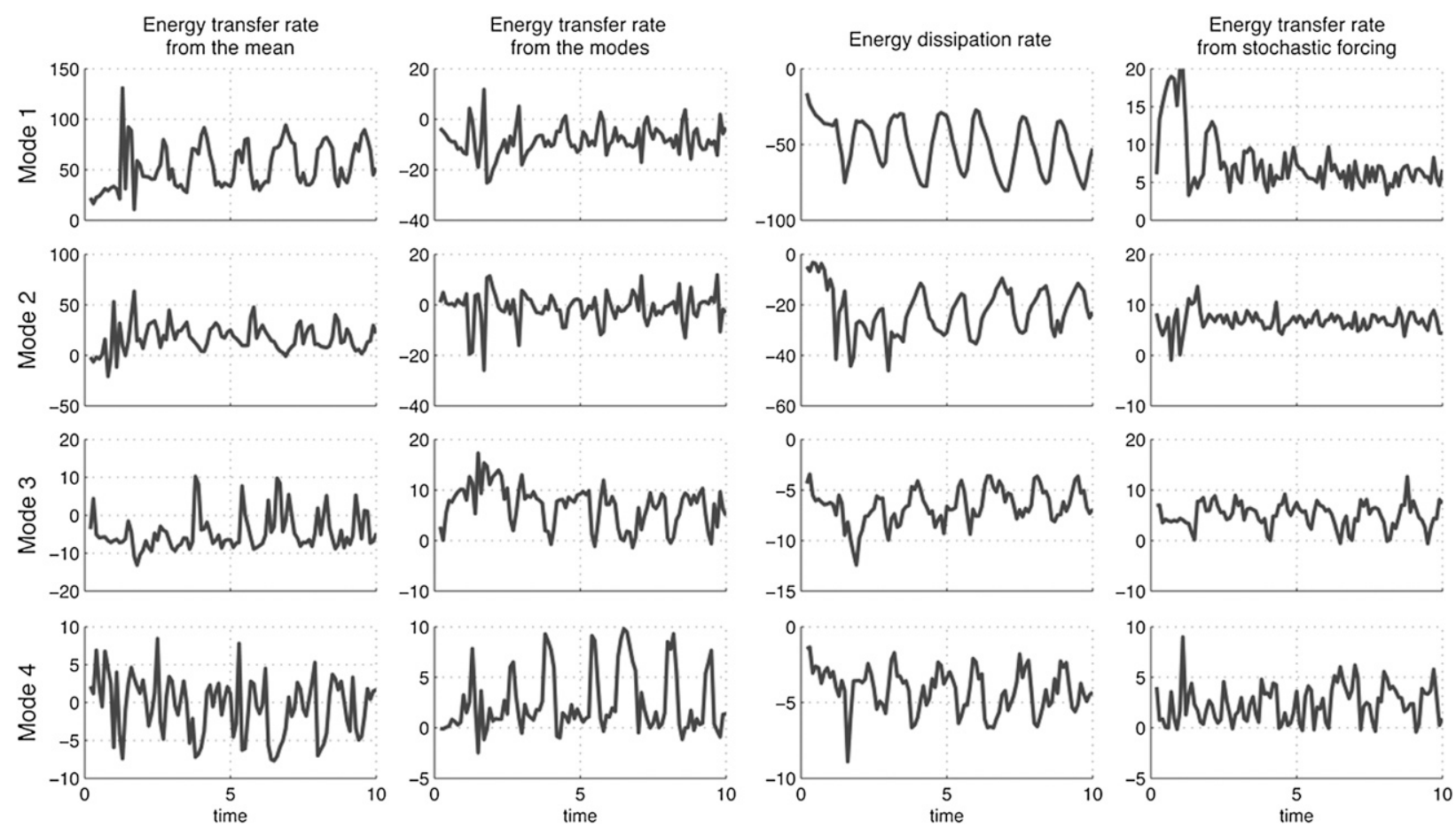

FIG. 9. Energy transfer rates for the case of temporally and spatially correlated stochastic excitation with decorrelation time scale $\tau=0.01$ and $\lambda=1 / 8$.

with stochastic coefficients, the latter satisfying a stochastic ordinary differential equation. From the amplitude of the mean, the DO-modes and the stochastic coefficients, the flow solution can be determined.

The bifurcation diagram of the deterministic case indicates different dynamical regimes, which are separated by specific bifurcation points (Dijkstra 2005). These different regimes are also found in the DO solutions for the case without noise and can be distinguished by the saturation of their amplitude in time. For example, the pitchfork $P_{1}$ distinguishes the regime where all DO modes decay in time from the regime where at least one of them grows to finite amplitude.

When spatially coherent but temporally white noise is added to the mean wind stress, we found that the DO mode patterns do not depend on the spatial structure of the noise. The analysis in section $3 b$ has shown that there is no exchange of energy from the stochastic excitation to the DO modes. From these results and theoretical arguments presented we may conclude that temporally uncorrelated additive noise has minimal effect on the reduced order dynamics of the system (both their statistics and their form, i.e., the basis elements) and the only effects observed are time-shifted transition behavior. This is consistent with the simulations in Pierini (2010), where no coherence resonance is found under white-noise wind forcing. The white-noise will also lead to an enhancement of probability spread around the chaotic attractor. This explains why in Sura et al. (2001), the noise is found to be important to induce transitions between different equilibrium states.

When there is memory in the noise, here induced by the temporal coherence in the wind stress forcing, modes take up energy from the stochastic excitation and there is a strong effect of the noise on the reduced dynamics and the energy transfers. Depending on the memory of the excitation, the system may be stabilized or destabilized. In the cases studied in Pierini (2010), coherence resonance occurs only when the decorrelation time scale is long enough and in his shallow-water model, it leads to more complex behavior. However, noise may also push the system into a completely different attractor with much less complex behavior characterized only by one unstable mode. This result is remarkable as it indicates that noise may induce relatively coherent behavior, resembling low-order behavior of the deterministic system.

The idealized configuration and model used here are obviously too simple to make any statement on the behavior of more complex ocean models and observations. The results should be seen as a first but important step in the study of the interaction of noise and nonlinear dynamics in spatially extended systems described by stochastic partial differential equations. As it has been 
shown that in the deterministic case, much of the behavior of solutions of the barotropic DG models is found in more complex models, such as shallow water equations (Pierini 2006; Pierini et al. 2009), we anticipate also that the effects of noise on the solutions may be similar, making the study here potentially relevant to understand the dynamics of midlatitude western boundary currents in the ocean.

Acknowledgments. This work was partly sponsored (HAD) by the COMPLEXITY project "Predictability of the Kuroshio current path transitions," which was funded by the Netherlands Organization for Scientific Research (NWO). We thank one of the anonymous referees for excellent comments, which have led to an improvement of the paper.

\section{APPENDIX}

\section{DO Equations for the DG Problem}

When the horizontal velocity is scaled with the scale $U$, length with $L$, time with $L / U$ and the pressure with $\rho_{0} U^{2}$, the dimensionless Eq. (4) become

$$
\begin{aligned}
\frac{\partial \mathbf{v}}{\partial t}= & -\nabla p+\frac{1}{\operatorname{Re}} \nabla^{2} \mathbf{v}-\mathbf{v} \cdot \nabla \mathbf{v}-f \mathbf{k} \times \mathbf{v}+\tau_{\mathbf{d}}(\mathbf{x}, t) \\
& +\sum_{k=1}^{s_{e}} Z_{k}(t ; \omega) \sigma_{k}(\mathbf{x}, t) \\
\nabla \cdot \mathbf{v}= & 0,
\end{aligned}
$$

where $\mathbf{v}=\left[v_{x}(\mathbf{x}, t ; \omega), v_{y}(\mathbf{x}, t ; \omega)\right]$ is the flow velocity field, and $\mathbf{k}$ is the unit vector in the $z$ direction. The pressure field is denoted with $p(\mathbf{x}, t ; \omega), f=\bar{f}+y$ (with $\bar{f}=f_{0} L^{2} \rho_{0} H \beta_{0} / \tau_{0}$ being irrelevant for the vorticity dynamics) is the Coriolis parameter under the $\beta$-plane approximation, $\tau_{d}(\mathbf{x}, t)=\left[\tau_{d}^{x}(\mathbf{x}, t), \tau_{d}^{y}(\mathbf{x}, t)\right]$ is the external deterministic stress acting on the fluid, $Z_{k}(t ; \omega)$ are scalar stochastic processes, and $\sigma_{k}(\mathbf{x}, t)=\left[\sigma_{k, x}(\mathbf{x}, t)\right.$, $\left.\sigma_{k, y}(\mathbf{x}, t)\right]$ is the zero-mean stochastic component of the wind stress for which we assume known complete probabilistic information.

In what follows we will use the inner product

$$
\langle\mathbf{v}, \mathbf{w}\rangle=\int_{V}\left(v_{x} w_{x}+v_{y} w_{y}\right) d \mathbf{x} .
$$

where $V$ is the volume of the ocean basin. We assume that the boundary conditions for the velocity vector $\mathbf{v}$ and the pressure are described by the linear differential operator $\mathcal{B}$

$$
\begin{aligned}
\mathcal{B}_{v}[\mathbf{v}(\xi, t ; \omega)] & =\mathbf{v}_{\partial V}(\xi, t), \\
\mathcal{B}_{p}[p(\xi, t ; \omega)] & =p_{\partial V}(\xi, t), \quad \xi \in \partial V .
\end{aligned}
$$

We also assume that the initial conditions are stochastic with known statistics given by

$$
\mathbf{v}\left(\mathbf{x}, t_{0} ; \omega\right)=\mathbf{v}_{0}(\mathbf{x} ; \omega), \quad \mathbf{x} \in V, \quad \omega \in \Omega .
$$

As state vector $\mathbf{u}$, we now take the velocity field $\mathbf{v}$ and by using a DO representation for the solution

$$
\mathbf{u}(\mathbf{x}, t ; \omega)=\overline{\mathbf{u}}(\mathbf{x}, t)+\sum_{i=1}^{s} Y_{i}(t ; \omega) \mathbf{u}_{i}(\mathbf{x}, t)
$$

we obtain the following set of equations.

- Equation for the mean field

$$
\begin{aligned}
\frac{\partial \overline{\mathbf{u}}}{\partial t}= & -\nabla p_{0}+\frac{1}{\operatorname{Re}} \nabla^{2} \overline{\mathbf{u}}-\overline{\mathbf{u}} \cdot \nabla \overline{\mathbf{u}}-f \mathbf{k} \times \overline{\mathbf{u}}+\tau_{d}(\mathbf{x}, t) \\
& -\mathbf{C}_{Y_{i}(t) Y_{j}(t)}\left(-\nabla p_{i j}+\frac{1}{2} \mathbf{u}_{i} \cdot \nabla \mathbf{u}_{j}+\frac{1}{2} \mathbf{u}_{j} \cdot \nabla \mathbf{u}_{i}\right) \\
0= & \nabla \cdot \overline{\mathbf{u}},
\end{aligned}
$$

where $\mathbf{C}$ indicates the covariance matrix and $p_{i j}$ is defined below.

- Equation for the stochastic coefficients

$$
\frac{d Y_{i}}{d t}=A_{i m}(t) Y_{m}+B_{i m n}(t) Y_{m} Y_{n}+D_{i}(t ; \omega),
$$

where

$$
\begin{aligned}
A_{i m}(t) & =\left\langle\frac{1}{\operatorname{Re}} \nabla^{2} \mathbf{u}_{m}-\mathbf{u}_{m} \cdot \nabla \overline{\mathbf{u}}-\overline{\mathbf{u}} \cdot \nabla u_{m}-f \mathbf{k} \times \mathbf{u}_{m}, \mathbf{u}_{i}\right\rangle \\
B_{i m n}(t) & =-\frac{1}{2}\left\langle\mathbf{u}_{m} \cdot \nabla \mathbf{u}_{n}+\mathbf{u}_{n} \cdot \nabla \mathbf{u}_{m}, \mathbf{u}_{i}\right\rangle, \\
D_{i}(t ; \omega) & =-B_{i m n}(t) \mathbf{C}_{Y_{m}(t) Y_{n}(t)}+\left\langle\sigma_{r}(\mathbf{x}, t), \mathbf{u}_{i}\right\rangle Z_{r}(t ; \omega) .
\end{aligned}
$$

- Equation for the DO modes

$$
\frac{\partial \mathbf{u}_{i}}{\partial t}=\mathbf{Q}_{\mathbf{u}, i}-\left\langle\mathbf{Q}_{\mathbf{u}, i}, \mathbf{u}_{m}\right\rangle \mathbf{u}_{m}, \quad \boldsymbol{\nabla} \cdot \mathbf{u}_{i}=0,
$$

where

$$
\begin{aligned}
\mathbf{Q}_{\mathbf{u}, i} \equiv & -\nabla p_{i}+\frac{1}{\operatorname{Re}} \nabla^{2} \mathbf{u}_{i}-\mathbf{u}_{i} \cdot \nabla \overline{\mathbf{u}}-\overline{\mathbf{u}} \cdot \nabla \mathbf{u}_{i}-f \mathbf{k} \times \mathbf{u}_{i}-\mathbf{C}_{Y_{i}(t) Y_{j}(t)}^{-1} \mathbf{M}_{Y_{j}(t) Y_{m}(t) Y_{n}(t)} \\
& \times\left(-\nabla p_{m n}+\frac{1}{2} \mathbf{u}_{m} \cdot \nabla \mathbf{u}_{n}+\frac{1}{2} \mathbf{u}_{n} \cdot \nabla \mathbf{u}_{m}\right)+\mathbf{C}_{Y_{i}(t) Y_{j}(t)}^{-1} \mathbf{C}_{Y_{j}(t) Z_{r}(t)}\left[-\nabla b_{r}+\sigma_{r}(\mathbf{x}, t)\right],
\end{aligned}
$$


- Accompanied with the pressure equations

$$
\begin{aligned}
\nabla^{2} p_{0} & =\nabla \cdot\left[-\overline{\mathbf{u}} \cdot \nabla \overline{\mathbf{u}}-f \mathbf{k} \times \overline{\mathbf{u}}+\tau_{d}(\mathbf{x}, t)\right] \\
\nabla^{2} p_{i} & =\nabla \cdot\left(-\mathbf{u}_{i} \cdot \nabla \overline{\mathbf{u}}-\overline{\mathbf{u}} \cdot \nabla \mathbf{u}_{i}-f \hat{\mathbf{k}} \times \mathbf{u}_{i}\right), \quad i=1, \ldots, s \\
\nabla^{2} p_{i j} & =\frac{1}{2} \nabla \cdot\left(\mathbf{u}_{i} \cdot \nabla \mathbf{u}_{j}+\mathbf{u}_{j} \cdot \nabla \mathbf{u}_{i}\right), \quad i=1, \ldots, s, \quad j=1, \ldots, s \\
\nabla^{2} b_{r} & =\nabla \cdot \sigma_{r}(\mathbf{x}, t), \quad k=1, \ldots, s .
\end{aligned}
$$

All these equations are accompanied with appropriate boundary conditions, which are described in full detail in Sapsis (2010). The numerical solution is performed using a finite-volume framework described in Ueckermann et al. (2013) that has been suitably modified to take into account the stochastic excitations described in this work.

\section{REFERENCES}

Cessi, P., and G. R. Ierley, 1995: Symmetry-breaking multiple equilibria in quasigeostrophic, wind-driven flows. J. Phys. Oceanogr., 25, 1196-1205.

Dijkstra, H. A., 2005: Nonlinear Physical Oceanography: A Dynamical Systems Approach to the Large Scale Ocean Circulation and El Niño. 2nd ed. Springer, 532 pp.

— the wind-driven ocean circulation. Geophys. Astrophys. Fluid Dyn., 83, 1-31.

— driven quasi-geostrophic double gyre ocean circulation: Basic bifurcation diagrams. Geophys. Astrophys. Fluid Dyn., 85, 195-232.

Gardiner, C. W., 2002: Handbook of Stochastic Methods. 2nd ed. Springer, $415 \mathrm{pp}$.

Gitterman, M., 2005: The Noisy Oscillator: The First Hundred Years, From Einstein until Now. World Scientific, 160 pp.
Henderson, D., and P. Plaschko, 2006: Stochastic Differential Equations in Science and Engineering. World Scientific, 240 pp.

Pedlosky, J., 1987: Geophysical Fluid Dynamics. 2nd ed. SpringerVerlag, 710 pp.

Pierini, S., 2006: A Kuroshio Extension system model study: Decadal chaotic self-sustained oscillations. J. Phys. Oceanogr., 36, 1605-1620.

2010: Coherence resonance in a double-gyre model of the Kuroshio Extension. J. Phys. Oceanogr., 40, 238-248.

— H. A. Dijkstra, and A. Riccio, 2009: A nonlinear theory of the Kuroshio Extension bimodality. J. Phys. Oceanogr., 39, 2212-2229.

Sapsis, T. P., 2010: Dynamically orthogonal field equations for stochastic fluid flows and particle dynamics. Ph.D. thesis, MIT, $252 \mathrm{pp}$.

— equations for continuous stochastic dynamical systems. Physica D, 238 (23-24), 2347-2360.

— and - 2012: Dynamical criteria for the evolution of the stochastic dimensionality in flows with uncertainty. Physica $D$, 241, 60-76.

Schmeits, M. J., and H. A. Dijkstra, 2001: Bimodality of the Kuroshio and the Gulf Stream. J. Phys. Oceanogr., 31, 2971-2985.

Simonnet, E., and H. A. Dijkstra, 2002: Spontaneous generation of low-frequency modes of variability in the wind-driven ocean circulation. J. Phys. Oceanogr., 32, 1747-1762.

Sura, P., 2003: Stochastic analysis of Southern and Pacific Ocean sea surface winds. J. Atmos. Sci., 60, 654-666.

— , and C. Penland, 2002: Sensitivity of a double-gyre ocean model to details of stochastic forcing. Ocean Modell., 4 (3-4), 327-345.

— F. Funkeit, and K. Fraedrich, 2000: Decadal variability in a simplified wind-driven ocean model. J. Phys. Oceanogr., 30, 1917-1930.

— K. Fraedrich, and F. Lunkeit, 2001: Regime transitions in a stochastically forced double-gyre model. J. Phys. Oceanogr., 31, 411-426.

Ueckermann, M. P., T. P. Sapsis, and P. F. J. Lermusiaux, 2013: Numerical schemes for dynamically orthogonal equations of stochastic fluid and ocean flows. J. Comput. Phys., 233, 272-294. 